\title{
DIAGNOSTIC CRITERIA FOR RHEUMATOID ARTHRITIS 1958 REVISION
}

\author{
BY

\section{A COMMITTEE OF THE AMERICAN RHEUMATISM ASSOCIATION}

(Chairman: DR. M. W. ROPES)

The criteria proposed for diagnostic classification of patients with rheumatoid arthritis have been in use for two years (Ropes, Bennett, Cobb, Jacox, and Jessar, 1956, 1957). During this period many comments have been received, the majority of which have been favourable. There have been, however, criticisms and suggestions which have formed the basis of this revision of the original criteria.

It has become apparent that the criteria are not quite rigorous enough. There has been rather general agreement that in addition to the three present groups there should be a group with even more strict criteria. In such a group the diagnosis of rheumatoid arthritis will be as certain as is possible with our present knowledge about and concept of the disease. The requirements for classification in this group are seven of the eleven criteria listed under the present heading of "Definite Rheumatoid Arthritis", and the new group is to be called "Classical Rheumatoid Arthritis". The significance of the other groups will remain unchanged. In the definite group there should be almost no question that every patient has rheumatoid arthritis, but in the new group more evidence for the diagnosis will usually be available in the form of $x$-ray changes, nodules, "rheumatoid factor", changes in synovial mucin, or characteristic histological findings.

Another point which has caused some discussion is the question of the duration of joint symptoms. The earlier criteria did not state whether the total duration of 6 weeks must be continuous, and whether each one of the symptoms must have been of 6 weeks' duration. The revised criteria require that the joint signs or symptoms in Criteria 1 to 5 must be continuous for at least 6 weeks in the Classical and Definite groups. In the case of the "Probable Rheumatoid Arthritis" group, the required duration is changed from 4 weeks to 6 weeks to lessen the confusion that has arisen in using different durations for the Definite and Probable groups. In only one of the Criteria 1 to 5 , however, need the signs or symptoms last the entire 6 weeks to qualify for the Probable group.

A question has arisen whether the criteria necessary for classification into any group must all be present simultaneously. It is our impression that usually the manifestations would all be present at the same time, but we do not consider such a situation necessary for placing a patient into any group.

Some difficulty was encountered in deciding which tests for the so-called "rheumatoid factor" should be accepted for Criterion 8. Many methods are being used and the relative diagnostic value of the various tests is not yet known. We suggest that any method may be used for demonstration of the rheumatoid factor that has been proved by at least two laboratories to be positive in not over 5 per cent. of normal controls.

The other questions that have arisen have been concerned with the "Exclusions". One more exclusion should be added: No. 20 "Agammaglobulinaemia". In this way the poorly understood rheumatic disease that occurs in patients with little or no gamma globulin will be excluded. The "L.E. cell" exclusion, No. 2, offers a more complicated problem, as the reported incidence increases of lupus erythematosus cells in patients with typical clinical pictures of rheumatoid arthritis and no characteristics (such as rash or renal involvement) strongly suggestive of disseminated lupus erythematosus. In the revised criteria, patients with a high concentration of lupus erythematosus cells (as in Exclusion 2) are still to be excluded from the rheumatoid categories, but are to be listed separately and discussed simultaneously with rheumatoid patients if they satisfy the present criteria and exclusions in every respect except Exclusion 2. In regard to the exclusion of gout, it should be pointed out that an increased level of uric acid in the blood does not necessarily exclude a diagnosis of rheumatoid arthritis. 


\section{REVISED CRITERIA}

\section{Classical Rheumatoid Arthritis}

This diagnosis requires seven of the following criteria. In Criteria 1 to 5 the joint signs or symptoms must be continuous for at least 6 weeks. (Any one of the features listed under "ExCLusions" will exclude a patient from this category.)

(1) Morning stiffness.

(2) Pain on motion or tenderness in at least one joint (observed by a physician).

(3) Swelling (soft tissue thickening or fluid-not bony overgrowth alone) in at least one joint (observed by a physician).

(4) Swelling (observed by a physician) of at least one other joint (any interval free of joint symptoms between the two joint involvements may not be more than 3 months).

(5) Symmetrical joint swelling (observed by a physician) with simultaneous involvement of the same joint on both sides of the body (bilateral involvement of mid-phalangeal, metacarpophalangeal, or metatarsophalangeal joints is acceptable without absolute symmetry). Terminal phalangeal joint involvement will not satisfy this criterion.

(6) Subcutaneous nodules (observed by a physician) over bony prominences, on extensor surfaces, or in juxta-articular regions.

(7) $X$-ray changes typical of rheumatoid arthritis (which must include at least bony decalcification localized to or greatest around the involved joints and not just degenerative changes)-degenerative changes do not exclude patients from any group classified as rheumatoid arthritis.

(8) Positive agglutination test-demonstration of the "rheumatoid factor" by any method that, in two laboratories, has been positive in not more than 5 per cent. of normal controls; or positive streptococcal agglutination test.

(9) Poor mucin precipitate from synovial fluid (with shreds and cloudy solution).

(10) Characteristic histological changes in synovial membrane with three or more of the following: marked villous hypertrophy; proliferation of superficial synovial cells often with palisading; marked infiltration of chronic inflammatory cells (lymphocytes or plasma cells predominating) with tendency to form "lymphoid nodules"; deposition of compact fibrin, either on surface or interstitially; foci of cell necrosis.

(11) Characteristic histological changes in nodules showing granulomatous foci with central zones of cell necrosis, surrounded by proliferated fixed cells, and peripheral fibrosis and chronic inflammatory cell infiltration, predominantly perivascular.

\section{Definite Rheumatoid Arthritis}

This diagnosis requires five of the above criteria. In Criteria 1 to 5 the joint signs or symptoms must be continuous for at least 6 weeks. (Any one of the features listed under "Exclusions" will exclude a patient from this category.)

\section{Probable Rheumatoid Arthritis}

This diagnosis requires three of the above criteria. In at least one of the Criteria 1 to 5 the joint signs or symptoms must be continuous for at least 6 weeks. (Any one of the features listed under "Exclusions" will exclude a patient from this category.)

\section{Possible Rheumatoid Arthritis}

This diagnosis requires two of the following criteria, $\vec{\infty}$ and the total duration of joint symptoms must be at least 3 weeks. (Any one of the features listed under $\overrightarrow{0}$ "Exclusions" will exclude a patient from this category.) 윽

(1) Morning stiffness.

(2) Tenderness or pain on motion (observed by a physician) with history of recurrence or persistence for 3 weeks.

(3) History or observation of joint swelling.

(4) Subcutaneous nodules (observed by a phy- Чٓ sician).

(5) Raised erythrocyte sedimentation rate or Creactive protein.

(6) Iritis.

\section{Exclusions}

(1) The typical rash of disseminated lupus erythematosus, with butterfly distribution, follicle plugging, 윽 and areas of atrophy.

(2) High concentration of lupus erythematosus cells (four or more in two smears prepared from? heparinized blood incubated for not more than 2 hrs).

(3) Histological evidence of periarteritis nodosa, $\overline{0}$ with segmental necrosis of arteries associated with nodular leucocytic infiltration extending perivascu- $\delta$ larly and tending to include many eosinophils.

(4) Weakness of neck, trunk, and pharyngeal muscles, or persistent muscle swelling of dermatomyositis.

(5) Definite scleroderma (not limited to the 을. fingers).

(6) A clinical picture characteristic of rheumatic fever, with migratory joint involvement and evidence 0 of endocarditis, especially if accompanied by sub- N cutaneous nodules or erythema marginatum or chorea. (A raised antistreptolysin titre will not rule out the diagnosis of rheumatoid arthritis.)

(7) A clinical picture characteristic of gouty arthritis, with acute attacks of swelling, redness, and $\stackrel{\oplus}{+}$ pain in one or more joints, especially if relieved by colchicine.

(8) Tophi.

(9) A clinical picture characteristic of acute $\frac{\mathscr{D}}{\mathbb{D}}$ infectious arthritis of bacterial or viral origin with 
an acute focus of infection or in close association with a disease of known infectious origin; chills; fever; an acute joint involvement, usually migratory initially (especially if there are organisms in the joint fluid or response to antibiotic therapy).

(10) Tubercle hacilli in joints or histological evidence of joint tuberculosis.

(11) A clinical picture characteristic of Reiter's syndrome, with urethritis and conjunctivitis associated with acute joint involvement, usually migratory initially.

(12) A clinical picture characteristic of the shoulder-hand syndrome, with unilateral involvement of shoulder and hand, with diffuse swelling of the hand followed by atrophy and contractures.

(13) A clinical picture characteristic of hypertrophic pulmonary osteo-arthropathy, with clubbing of fingers and'or hypertrophic periostitis along the shafts of the long bones especially if an intrapulmonary lesion is present.

(14) A clinical picture characteristic of neuroarthropathy, with condensation and destruction of bones of involved joints and with associated neurological findings.

(15) Homogentisic acid in the urine detectable grossly with alkalinization.

(16) Histological evidence of sarcoid or a positive Kveim test.

(17) Multiple myeloma as evidenced by marked increase in plasma cells in the bone marrow or by Bence-Jones protein in the urine.

(18) Characteristic skin lesions of erythema nodosum.

(19) Leukaemia or lymphoma, with characteristic cells in peripheral blood, bone marrow, or tissues.

(20) Agammaglobulinaemia.

It is hoped that these revised criteria will aid in obtaining even more uniformity in the classification of patients with rheumatoid arthritis. Comments and suggestions for modifications or clarification will be welcomed. The criteria should be reviewed in two or three years and revised as necessary.

\section{REFERENCES}

Ropes, M. W., Bennett, G. A., Cobb, S., Jacox, R., and Jessar, R. A. (1956). Bull. rheum. Dis., 7, 121.

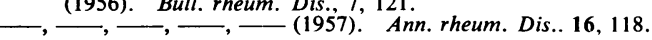

\section{Critères diagnostiques pour l'arthrite rhumatismale. Revision, 1958}

\section{Arthrite Rhumatismale Classique}

Ce diagnostic nécessite sept des critères énumérés ci-dessous. Pour satisfaire les critères 1 à 5 les signes et les symptômes articulaires doivent être continus depuis au moins six semaines. (L'un quelconque des caractères énumérés sous le titre "ExCLuSIONS" exclura le malade de cette catégorie).

1. Enraidissement matinal.

2. Mouvement douloureux ou sensibilité dans une articulation au moins (observés par un médecin).
3. Tuméfaction (observée par un médecin) d'une articulation au moins (épaississement du tissu mou ou excès de liquide et non pas excroissance osseuse seule).

4. Tuméfaction (observée par un médecin) d'au moins une autre articulation (toute période de temps sans symptômes articulaires, s'écoulant entre les implications des deux articulations, ne doit pas dépasser trois mois).

5. Tuméfaction articulaire symétrique (observée par un médecin) avec atteinte simultanée de la même articulation de deux cotés du corps (l'atteinte bilatérale des articulations mi-phalangiennes, métacarpo-phalangiennes ou métatarso-phalangiennes est acceptable sand symétrie absolue). L'atteinte de l'articulation phalangienne terminale ne satisfait pas ce critère.

6. Nodules sous-cutanés (observés par un médecin) sur des proéminences osseuses, dans la région des extenseurs ou juxta-articulaires.

7. Altérations radiologiques typiques d'arthrite rhumatismale (devant comprendre au moins une décalcification osseuse localisée ou prédominante aux articulations atteintes et non pas des signes de dégénérescence seuls)-les signes de dégénérescence n'excluent pas les malades du groupe d'arthrite rhumatismale.

8. Réaction d'agglutination positive-mise en evidence du "facteur rhumatismal" par une méthode quelconque qui, dans deux laboratoires, n'a donné plus de 5 pour cent de résultats positifs chez des témoins normaux; ou réaction d'agglutination streptococcique positive.

9. Faible précipité de mucine du liquide synovial (avec fibres et solution trouble).

10. Altérations histologiques caractéristiques de la membrane synoviale, dont trois ou plus des suivantes: hypertrophie villeuse marquée; prolifération des cellules synoviales superficielles, souvent avec palissage; infiltration marquée par éléments inflammatoires chroniques (lymphocytes ou éléments plasmatiques prédominant) avec tendance à former des "nodules lymphoïdes"; depôt de fibrine compacte soit en surface, soit interstitiellement; foyers de nécrose cellulaire.

11. Altérations histologiques caractéristiques des nodules, comprenant des foyers granulomateux avec zones centrales de nécrose cellulaire, entourées de cellules proliférantes fixes, de fibrose périphérique et d'infiltration cellulaire inflammatoire chronique essentiellement périvasculaire.

\section{Arthrite Rhumatismale Definie}

Ce diagnostic nécessite cinq des critères énumérés ci-dessus. Dans les critères 1 à 5 les signes et les symptômes articulaires doivent êtres continus pendant au moins six semaines. (L'un quelconque des caractères énumérés sous le titre "Exclusions" exclura le malade de cette catégprie.)

\section{Arthrite Rhumatismale Probable}

Ce diagnostic nécessite trois des critères énumérés ci-dessus. Dans un au moins des critères 1 à 5 les signes et les symptômes articulaires doivent être continus pendant au moins six semaines. (L'un quelconque des 
caractères énumérés souls le titre "ExcLusions" exclura le malade de cette catégorie.)

\section{Arthrite Rhumatismale Possible}

Ce diagnostic nécessite deux des critères suivants, et la durée totale des symptômes articulaires doit être au moins de trois semaines. (L'un quelconque des caractères énumérés sous le titre "ExcLusions" exclura le malade de cette catégorie):

1. Enraidissement matinal.

2. Sensibilité ou mouvement douloureux (observés par un médecin) avec antécédants de récurrence ou de persistance pendant trois semaines.

3. Antécédents ou constatation de tuméfaction articulaire.

4. Nodules sous-cutanés (observés par un médecin).

5. Vitesse de sédimentation érythrocytaire élevée ou présence de protéine C-réactive.

6. Irite.

\section{ExCLUSIONS}

1. L'éruption typique de lupus érythémateux disséminé (avec répartition en papillon, obturation folliculaire et plaques d'atrophie).

2. Forte concentration de cellules de lupus érythémateux (quatre ou plus, en deux frottis préparés avec du sang héparinisé, incubés pas plus de deux heures).

3. Signes histologiques de périartérite noueuse avec nécrose segmentaire des artères, associée à une infiltration nodulaire leukocytaire, s'étendant périvasculairement et tendant à inclure de nombreux éosinophiles.

4. Faiblesse des muscles du cou, du tronc et du pharynx ou enflure musculaire persistente de la dermatomyosite.

5. Sclérodermie établie (non limitée aux doigts).

6. Tableau clinique caractéristique de rhumatisme polyarticulaire aigu avec implication articulaire migrante et signes d'endocardite, surtout en présence de nodules sous-cutanés, d'érythème marginé ou de chorée. (Un titre élevé d'antistreptolysine n'éliminera pas le diagnostic d'arthrite rhumatismale.)

7. Tableau clinique caractéristique d'arthrite goutteuse avec attaques aigus de tuméfaction, rougeur et douleur surtout soulagés par la colchicine.

8. Tophi.

9. Tableau clinique caractéristique d'arthrite infectieuse aiguë d'origine bactérienne ou à virus, avec un foyer aigu d'infection ou en association étroite avec une maladie d'origine infectieuse connue; rhumes; fièvre; atteinte articulaire aiguë, d'abord habituellement migrante (en particulier si l'on trouve des organismes dans le liquide synovial ou si la réponse à la thérapie antibiotique est favorable).

10. Bacilles de Koch dans les articulations ou signes histologiques de tuberculose articulaire.

11. Tableau clinique caractéristique de syndrome de Reiter, avec urethrite et conjonctivite associées à l'atteinte articulaire aiguë, habituellement migrante au début.
12. Tableau clinique caractéristique de syndrome épaule-main, avec atteinte unilatérale de l'épaule é et de la main et tuméfaction diffuse de la main; suivie d'atrophie et de contractures.

13. Tableau clinique caractéristique d'ostéoarthropathie pulmonaire hypertrophique avec doigts noueux et/ot? périostite hypertrophique diaphysaire des os longs은 en particulier en présence d'une lésion intra $\frac{\sqrt{5}}{7}$ pulmonaire.

14. Tableau clinique caractéristique de neuro-arthro pathie avec condensation et destruction osseuse deథs articulations impliquées et signes neurologiques associés.

15. Acide homogentisique dans l'urine, décélable्ట్ grossièrement par alcalinisation.

16. Signes histologiques de sarcoïde ou épreuve positiv de Kveim.

17. Myelome multiple mis en évidence par un accroisse ment marqué des éléments plasmatiques dans la moelle osseuse, ou présence de la protéine dế Bence-Jones dans l'urine.

18. Lésions cutanées caractéristiques de l'érythème nоuеux.

19. Leucémie ou lymphome avec éléments caractéris tiques dans le sang périphérique, la moelle osseuse ou les tissus.

20. Agammaglobulinémie.

Criterios diagnósticos para la artritis reumatoide. Revisión, 1958

Artritis Rheumatoide Clasica

Este diagnóstico necesita siete de los criterios siguientes尺ి Para satisfacer los criterios de 1 a 5 los signos y sintoma articulares deben ser continuos durante seis semanas a $\vec{b}$ menos. (Cualquiera de los rasgos enumerados bajo e $\$$ título "ExClusiones" excluye el enfermo de esta cate goría.)

1. Rigidez matinal.

2. Movimiento doloroso o sensibilidad en una articulación al menos (observados por un médico)

3. Hinchazón (observada por un médico) de unå․ articulación al menos (espesamiento de tejidch blando o líquido, pero no acrecentamineto óseô solo).

4. Hinchazón (observada por un médico) de al menos una otra articulación (cualquier período de tiempo sin síntomas articulares entre las implicaciones de dos articulaciones no debe rebasar tres meses).

5. Hinchazón articular simétrica (observada por uro médico) con implicación simultánea de la mismă articulación de ambos lados del cuerpo (la implio cación bilateral de las articulaciones falangea medias, metacarpo-falangeas o metatarso-falangeas es aceptable sin simetría absoluta). La implicaciór de la articulación falangea terminal no satisface este criterio.

6. Nódulos subcutáneos (observados por un médico sobre prominencias óseas, en la región de lost extensores o yuxta-articulares.

7. Alteraciones radiológicas típicas de artritis reuma toide (que deben comprender al menos una decalci $\stackrel{\mathbb{D}}{\Omega}$ ficación ósea localizada o predominante en las 
articulaciones implicadas y no sólo alteraciones degenerativas) - alteraciones degenerativas no excluyen los casos del grupo de artritis reumatoide.

8. Reacción de aglutinación positiva-demostración del "factor reumatoide" por un método cualquiera que, en dos laboratorios, no ha dado más de un 5 por ciento de resultados positivos en testigos normales; o reacción de aglutinación estreptococcica positiva.

9. Precipitado débil de mucina del líquido sinovial (con trizas y solución turbia).

10. Alteraciones histológicas características de la membrana sinovial, con tres o más rasgos siguientes: hipertrofia vellosa acentuada; proliferación de las células sinoviales superficiales, a menudo con palizada; infiltración marcada por elementos inflamatorios crónicos (con linofocitos o elementos plasmáticos predominantes) con tendencia a formar "nódulos linfoides"; depósito de fibrina compacta superficial $o$ intersticialmente; focos de necrosis celular.

11. Alteraciones histológicas características en los nódulos, comprendiendo focos granulomatosos con zonas centrales de necrosis celular, rodeadas de células proliferantes fijas, y una fibrosis periférica así como infiltración celular inflamatoria crónica, esencialmente perivascular.

\section{Artritis Rheumatoide Definida}

Este diagnóstico necesita cinco de los criterios enumerados. Para satisfacer los criterios de 1 a 5 los signos y síntomas articulares deben ser continuos durante seis semanas al menos. (Cualquiera de los rasgos enumerados bajo el título "ExCLUSIONEs" excluye el enfermo de esta categoría.)

\section{Artritis Reumatoide Probable}

Este diagnóstico necesita tres de los criterios ya enumerados. Para satisfacer uno al menos de los criterios de 1 a 5 , los signos y los síntomas articulares deben ser continuos durante seis semanas al menos. (Cualquiera de los rasgos enumerados bajo el título "Exclusiones" excluye el enfermo de esta categoría.)

\section{Artritis Reumatoide Posible}

Este diagnóstico necesita dos de los criterios siguientes y la duración total de los síntomas articulares debe ser al menos de tres semanas (cualquiera de los rasgos enumerados bajo el título "ExCLUSIONEs" excluye el enfermo de esta categoría):

1. Rigidez matinal.

2. Sensibilidad o movimiento doloroso (observados por un médico) con antecedentes de recurrencia o persistencia durante tres semanas.

3. Antecedentes u observación de hinchazón articular.

4. Nódulos subcutáneos (observados por un médico).

5. Velocidad de sedimentación eritrocitaria aumentada o presencia de proteina C-reactiva.

6. Iritis.

\section{EXCLUSIONES}

1. Erupción típica de lupus eritematoso diseminado (con distribución en mariposa, obturación folicular y placas de atrofia).
2. Fuerte concentración de células de lupus eritematoso (cuatro o más, en dos frotis preparados con sangre heparinizada, incubados en menos de dos horas).

3. Signos histológicos de periarteritis nodosa con necrosis segmentaria de las arterias, asociada a una infiltración nodular leucocitaria con extensíon perivascular y con tendencia a incluir numerosos eosinófilos.

4. Debilidad muscular del cuello, del tronco y de la faringe o hinchazón muscular persistente de la dermatomiositis.

5. Esclerodermia establecida (no limitada a los dedos).

6. Cuadro clínico característico de reumatismo poliarticular agudo con implicación articular migratoria y signos de endocarditis, particularmente en presencia de nodulos subcutáneos, de eritema marginato o de corea. (Una cifra alta de antistreptolisina no excluye el diagnóstico de artritis reumatoide.)

7. Un cuadro clínico característico de artritis gotosa, con ataques agudos de hinchazón, rubor y dolor en una o más articulaciones, particularmente cuando la colchicina procura un alivio.

8. Tofos.

9. Cuadro clínico característico de artritis infecciosa aguda debida a bacterias o virus, con un foco agudo de infección o en asociación estrecha con una enfermedad de origen infeccioso conocido; resfríos: fiebre; y un ataque articular agudo, generalmente migratorio al principio (en particular, cuando se encuentran organismos en el líquido articular $o$ cuando hay respuesta a la terapia antibiótica).

10. Bacilos tuberculosos en las articulaciones o signos histológicos de tuberculosis articular.

11. Cuadro clínico característico de sindrome de Reiter, con uretritis y conjuntivitis, asociadas a un ataque articular agudo, generalmente migratorio al principio.

12. Cuadro clínico característico de sindrome hombromano, con implicación unilateral del hombro y de la mano, hinchazón difusa de la mano, seguidas de atrofia y de contracturas.

13. Cuadro clínico característico de osteo-artropatia pulmonar hipertrófica con dedos nodosos y/o periostitis hipertrófica diafisaria, en particular en presencia de una lesión intrapulmonar.

14. Cuadro clínico característico de neuroatropatia con condensación y destrucción ósea de las articulaciones implicadas y con signos neurológicos asociados.

15. Ácido homogentisico en la orina, detectable groseramente por alcalinización.

16. Signos histológicos de sarcoide o reacción positiva de Kveim.

17. Mieloma multiple, evidenciado por un acrecimiento marcado de elementos plasmáticos en la médula ósea o la proteina de Bence-Jones en la orina.

18. Lesiones cutaneas características de eritema nodoso.

19. Leucemia o linfoma con elementos característicos en la sangre periférica, la medula ósea o los tejidos.

20. Agamaglobulinemia. 